\title{
THE FRATTINI SUBALGEBRA OF A BERNSTEIN ALGEBRA*
}

\author{
by JESÜS LALIENA
}

(Received 8th May 1990)

Let $A$ be a finite-dimensional Bernstein algebra over a field $K$ with characteristic not 2. Maximal subalgebras of $A$ are studied, and they are determined if $A$ is a genetic aigebra. It is also proved that the intersection of all maximal subalgebras of $A$ (the Frattini subalgebra of $A$ ) is always an ideal. Finally the structure of Bernstein algebras with Frattini subalgebra equal to zero is described.

1980 Mathematics subject classification (1985 Revision). 17DXX.

\section{Introduction}

A finite-dimensional commutative algebra over a field $K$ is called baric is there exists a non trivial algebra homomorphism $w: A \rightarrow K$.

A baric algebra is said to be a Bernstein algebra if

$$
x^{2} \cdot x^{2}-(w(x))^{2} \cdot x^{2}=0 \text { for every } x \text { in } A .
$$

Bernstein algebras have connections with genetics (see $[2,3$, and 8$]$ ).

The homomorphism $w$ is called the weight homomorphism of $A$. In [8] it is shown that in a Bernstein algebra this homomorphism is unique.

In a Bernstein algebra $A$ there exists a nonzero idempotent $e$ and $A$ has a decomposition as a direct sum of vector subspaces (see [8]):

$$
A=K . e \oplus U_{e} \oplus V_{e},
$$

with $U_{e}=\{x \in \operatorname{Ker} w / e x=(1 / 2) x\}$ and $V_{e}=\{x \in \operatorname{Ker} w / e x=0\}$. This decomposition is called the Peirce decomposition of $A$. If we express the relation " $A$ is a vector subspace of $B$ " by $A \leqq B$, the vector subspaces $U_{e}, V_{e}$ have the following properties:

$$
U_{e}^{2} \leqq V_{e} \quad U_{e} V_{e} \leqq U_{e} \quad V_{e}^{2} \leqq U_{e} \quad V_{e}^{2} U_{e}=0
$$

and using (1) it is possible to prove that for all $u \in U_{e}$ and $v \in V_{e}$

$$
u^{3}=0 \quad u(u v)=0
$$

*Partially supported by D.G.A. CB 1/88. 


$$
\begin{aligned}
& u^{2}(u v)=0 \quad(u v)^{2}=0 \\
& u^{2} v^{2}=0
\end{aligned}
$$

In the above situation, the set of idempotents in $A$ is $\left\{e+u+u^{2} / u \in U_{e}\right\}$. If $e^{\prime}=e+u+u^{2}$ is another idempotent in $A$, we have $A=K . e^{\prime}+U_{e^{\prime}}+V_{e^{\prime}}$ and then $U_{e^{\prime}}=\left\{u_{1}+2 u u_{1} / u_{1} \in U_{e}\right\}$ and $V_{e^{\prime}}=\left\{-2\left(u+u^{2}\right) v_{1}+v_{1} / v_{1} \in V_{e}\right\}$.

A Bernstein algebra is called genetic if Ker $w$ is nilpotent, that is, if there exists a nonzero positive integer $n$ such that the principal product of every set of $n$ elements from $\operatorname{Ker} w$ is zero.

Let $A$ be an algebra and $M$ a subalgebra of $A$. $M$ is said to be a maximal subalgebra of $A$ if for every subalgebra $B$ of $A$, such that $M \leqq B \leqq A$, we have either $M=B$ or $B=A$. The intersection of all maximal subalgebras of $A$ is known as the Frattini subalgebra $F(A)$ of $A$ (see [6]). It has the following properties:

(P1) Let $C$ be a subalgebra of $A$ and $B$ an ideal of $A$ such that $B \leqq F(C)$. Then $B \leqq F(A)$.

(P2) (i) If $B$ is an ideal of $A$ we have $(F(A)+B) / B \leqq F(A / B)$.

(ii) Let $B$ be in ideal of $A$ such that $B \leqq F(A)$. Then $F(A) / B=F(A / B)$.

(P3) If $B$ is an ideal of $A$ such that $B^{2}=0$ and $B \cap \phi(A)=0$, with $\phi(A)$ the largest ideal of $A$ contained in $F(A)$, then there exists a subalgebra $C$ of $A$ such that $A=B \oplus C$. That is, $A$ is the direct sum of the vector subspaces $B$ and $C$.

(P4) If $A$ is a nilpotent finite-dimensional algebra, then $F(A)=A^{2}$.

In the following $A$ will always be a Bernstein algebra with $1<\operatorname{dim}_{K} A<\infty$, over a field $K$, with characteristic not 2. The weight homomorphism of $A$ will be denoted by $w$. If $X$ is a subset of $A$, we denote by $(X)$ the vector subspace of $A$ generated by $X$ and $\langle X\rangle$ the subalgebra of $A$ generated by $X$. Sometimes if $X$ has only one element, $a$, we also write $K . a$ instead of $(a)$.

\section{Maximal subalgebras of a Bernstein algebra}

From Theorem 1 in [1] we can deduce that every maximal subalgebra of a $n$-dimensional Bernstein algebra has dimension equal to $n-1$. This result will be very important in the following discussion.

Lemma 1. Let $A$ be a Bernstein algebra, $e$ a nonzero idempotent in $A$ such that $A=\operatorname{Ke} \oplus U_{e} \oplus V_{e}$, and $M$ a maximal suhalgebra of $A$. Then

(i) $U_{e}^{2} \leqq M$,

(ii) if $e \in M, V_{e}^{2} \leqq M$.

Proof. We suppose that $U_{e}^{2}$ is not contained in $M$. Thus, let $x \in U_{e}^{2}-M$. Since $\operatorname{dim} M+1=\operatorname{dim} A$ we have $M+K . x=A$ with $K . x \leqq V_{e}$. Therefore, $e=m+\tau x$ with $m \in M$ and $\tau \in K$. But since $A=\operatorname{Ke} \oplus U_{e} \oplus V_{e}$ it follows that $m=e-\tau x$, and hence 
$m^{2}=e \in M$. Now if $u \in U_{e}$, we have as before $u=m^{\prime}+\lambda x$ with $m^{\prime}=u-\lambda x \in M$ and $1 / 2 u \in M$. That is $u \in M$ for every $u \in U_{e}$. But this contradicts the fact that $U_{e}^{2}$ is not contained in $M$.

Now we suppose that $V_{e}^{2}$ is not contained in $M$. We consider $y \in V_{e}^{2}-M$ and thus $A=M \oplus K . y$ with $K . y \leqq U_{e}$. But if $e \in M$ we have $M=K . e \oplus U_{e}^{\prime} \oplus V_{e}^{\prime}$ with $U_{e}^{\prime} \leqq U_{e}$ and $V_{e}^{\prime} \leqq V_{e}$. Therefore $V_{e} \leqq M$. That is, $V_{e}^{2}$ is contained in $M$, which is a contradiction.

If $A$ is a Bernstein algebra and $B$ is a subalgebra of $A$ such that $B$ is not contained in Ker $w$, then $B$ has a nonzero idempotent $e$, because $B$ is also a Bernstein algebra.

Proposition 2. Let $A$ be a Bernstein algebra, and $e$ a nonzero idempotent in $A$ such that $A=\operatorname{Ke} \oplus U_{e} \oplus V_{e}$. Then a vector subspace of $A, M$, is a maximal subalgebra if and only if $M$ is one of the following subalgebras:

(i) $M=\operatorname{Ker} w$,

(ii) $M=K . e \oplus U_{e} \oplus V_{e}^{\prime}$ with $V_{e}^{\prime} \leqq V_{e}$ such that $\operatorname{dim} V_{e}+1=\operatorname{dim} V_{e}$ and $U_{e}^{2} \leqq V_{e}^{\prime}$. In this case $M$ is an ideal.

(iii) $M=K . e \oplus U_{e}^{\prime} \oplus V_{e}$ with $U_{e}^{\prime} \leqq U_{e}, \operatorname{dim} U_{e}^{\prime}+1=\operatorname{dim} U_{e}, U_{e}^{\prime} V_{e}+V_{e}^{2} \leqq U_{e}^{\prime}$,

(iv) $M=(e+u) \oplus U_{e}^{\prime} \oplus V_{e} \quad$ with $\quad U_{e}^{\prime} \leqq U_{e}, \quad \operatorname{dim} U_{e}^{\prime}+1=\operatorname{dim} U_{e}, \quad U_{e}^{\prime} V_{e}+V_{e}^{2} \leqq U_{e}^{\prime}$, $u \in U_{e}-U_{e}^{\prime}$

(v) $M=K . e_{M} \oplus U_{e_{M}}^{\prime} \oplus V_{e_{M}}$ with $e_{M}=e+u+u^{2}, u \notin M, V_{e}$ not contained in $M$ and $U_{e_{M}}^{\prime} \leqq U_{e_{M}}$ such that $\operatorname{dim} U_{e_{M}}^{\prime}+1=\operatorname{dim} U_{e_{M}}$ and $U_{e_{M}}^{\prime} V_{e_{M}}+U_{e_{M}}^{2} \leqq U_{e_{M}}^{\prime}$.

Proof. We suppose $M \neq \operatorname{Ker} w$. Thus $M$ contains an idempotent and $\left.w\right|_{M}$ is a nonzero homomorphism from $M$ onto $K$. Therefore $M /\left.\operatorname{Ker} w\right|_{M} \cong K$ and $\left.\operatorname{dim} \operatorname{Ker} w\right|_{M}$ $=\operatorname{dim} M-1$. Let $B=\operatorname{Ker} w \cap M=\left.\operatorname{Ker} w\right|_{M}$. We know that the set of idempotents in $A$ is $\left\{e+u+u^{2} / u \in U_{e}\right\}$. Let $e+u+u^{2} \in M$ with $u \in U_{e}$. Since $U_{e}^{2} \leqq M$ because of Proposition 1 , we have $e+u \in M$.

If $U_{e} \leqq M$, then $M=K . e \oplus U_{e} \oplus V_{e}^{\prime}$ with $V_{e}^{\prime} \leqq V_{e}$ such that $\operatorname{dim} V_{e}^{\prime}+1=\operatorname{dim} V_{e}$. Then $M$ contains every idempotent of $A$, and $M$ is an ideal because $A . M=$ $\left(K . e \oplus U_{e} \oplus V_{e}\right) .\left(K . e \oplus U_{e} \oplus V_{e}^{\prime}\right)=K . e \oplus U_{e} \oplus U_{e}^{2} \leqq M$. Thus we obtain (ii).

If $U_{e}$ is not contained in $M$ but $u \in M$, then $e \in M$ and $M=K . e \oplus B=K . e \oplus U_{e}^{\prime} \oplus V_{e}^{\prime}$ with $U_{e}^{\prime} \leqq U_{e}$ and $V_{e}^{\prime} \leqq V_{e}$. Since $\operatorname{dim} B+1=\operatorname{dim} \operatorname{Ker} w$, we have $M=K . e \oplus U_{e}^{\prime} \oplus V_{e}$. Also $U_{e}^{\prime} V_{e}+V_{e}^{2} \leqq U_{e}^{\prime}$ because $M$ is a subalgebra, and thus we obtain (iii).

If $V_{e}^{2} \leqq M$ and $u \notin M$ we will prove that $V_{e} \leqq M$. We have that $\operatorname{Ker} w=B \oplus K$.u. Let $v \in V_{e}$. Then $v=b+\lambda u$ with $b \in B$ and $\lambda \in K$. Thus $(e+u)(v-\lambda u)=u v-\lambda / 2 u-\lambda u^{2} \in B$ and $b^{2}=(v-\lambda u)^{2}=v^{2}+\lambda^{2} u^{2}-2 \lambda u v \in B$. Hence, since $U_{e}^{2}, V_{e}^{2} \leqq M$ because of Lemma 1 and the hypothesis, it follows that $\lambda u \in B$ and therefore $v \in M$. Thus $M=(e+u) \oplus U_{e}^{\prime} \oplus V_{e}$ with $U_{e}^{\prime} \leqq U_{e}$ such that $\operatorname{dim} U_{e}^{\prime}+1=\operatorname{dim} U_{e}, V_{e}^{2} \leqq U_{e}^{\prime}$ and $0 \neq u \in U_{e}-U_{e}^{\prime}$. Since $M$ is a subalgebra, it follows also that $U_{e}^{\prime} V_{e} \leqq U_{e}^{\prime}$ and we have (iv).

Now we suppose $u \notin M$ and $V_{e}$ is not contained in $M$. Then if $e_{M}=e+u+u^{2}$, it follows that $M=K . e_{M} \oplus U_{e_{M}}^{\prime} \oplus V_{e_{M}}^{\prime}$ with either $U_{e_{M}}^{\prime}=U_{e_{M}}$ or $V_{e_{M}}^{\prime}=V_{e_{M}}$. But if $U_{e_{M}}^{\prime}=U_{e_{M}}$ we have shown that $M$ contains every idempotent of $A$, that is $u \in M$ that contradicts the hypothesis. Therefore $M=K . e_{M} \oplus U_{e_{M}}^{\prime} \oplus V_{e_{M}}$ and as in (iii) it follows that $\operatorname{dim} U_{e_{M}}^{\prime}+1=\operatorname{dim} U_{e_{M}}$ and $U_{e_{M}}^{\prime} V_{e_{M}}+U_{e_{M}}^{2} \leqq U_{e_{M}}^{\prime}$. 
Lemma 3. Let $A$ be a genetic Bernstein algebra. Then $(\mathrm{Ker} w)^{2}$ is contained in every maximal subalgebra of $M$.

Proof. Since $A$ is genetic, Ker $w$ is nilpotent and thus from (P4) we have $F(\operatorname{Ker} w)=(\operatorname{Ker} w)^{2}$. Using that $(\operatorname{Ker} w)^{2}=U_{e}^{2}+U_{e} V_{e}+V_{e}^{2}$ we have that $(\operatorname{Ker} w)^{2}$ is an ideal. Hence from $(\mathrm{P} 1)$ we obtain $(\operatorname{Ker} w)^{2} \leqq F(A)$. That is, $(\operatorname{Ker} w)^{2} \leqq M$ for maximal subalgebra $M$ of $A$.

The result of Lemma 3 is not true if $A$ is only a Bernstein algebra. For instance the commutative algebra $A=(e, u, v, z)$ such that $e u=1 / 2 u, u v=u, e^{2}=e$ and the other products equals to zero is a Bernstein algebra, but the maximal subalgebra $(e, v, z)$ does not contain $(\operatorname{Ker} w)^{2}=(u, v, z)^{2}=(u)$.

However there are Bernstein algebras which are not genetic and for which $(\operatorname{Ker} w)^{2} \leqq$ $M$, for every maximal subalgebra $M$ of $A$. For example the commutative algebra $A=(e, u, v, z)$ with $e^{2}=e, e u=1 / 2 u, u v=u z=v z=u$ and the other products zero is a Bernstein algebra such that $(\operatorname{Ker} w)^{2}=(u)$ is contained in every maximal subalgebra.

Theorem 4. Let $A$ be a genetic Bernstein algebra and $e$ a nonzero idempotent in $A$ such that $A=\operatorname{Ke} \oplus U_{e} \oplus V_{e}$. Then a vector subspace $M$ of $A$, is a maximal subalgebra if and only if $M$ satisfies one of the following conditions:

(i) $M=\operatorname{Ker} w$,

(ii) $M=K . e \oplus U_{e} \oplus V_{e}^{\prime}$ with $V_{e}^{\prime} \leqq V_{e}$ such that $\operatorname{dim} V_{e}^{\prime}+1=\operatorname{dim} V_{e}$ and $U_{e}^{2} \leqq V_{e}^{\prime}$,

(iii) $M=K . e \oplus U_{e}^{\prime} \oplus V_{e}$ with $U_{e}^{\prime} \leqq U_{e}, \operatorname{dim} U_{e}^{\prime}+1=\operatorname{dim} U_{e}, U_{e} V_{e}+V_{e}^{2} \leqq U_{e}^{\prime}$,

(iv) $M=(e+u) \oplus U_{e}^{\prime} \oplus V_{e} \quad$ with $\quad U_{e}^{\prime} \leqq U_{e}, \quad \operatorname{dim} U_{e}^{\prime}+1=\operatorname{dim} U_{e}, \quad U_{e} V_{e}+V_{e}^{2} \leqq U_{e}^{\prime}$, $u \in U_{e}-U_{e}^{\prime}$.

Proof. From Lemma $3(\operatorname{Ker} w)^{2}=U_{e}^{2}+U_{e} V_{e}+V_{e}^{2}$ is contained in every maximal subalgebra, and from Proposition 2 and its proof we have that $M$ is as in (i), (ii), (iii) or (iv).

Corollary 5. If $A$ is a genetic Bernstein algebra, then $F(A)=(\operatorname{Ker} w)^{2}$.

\section{The Frattini subalgebra}

In this paragraph we study the intersection of all maximal subalgebras of a general Bernstein algebra, that is, its Frattini subalgebra. We also describe Bernstein algebras with Frattini subalgebra equal to zero, using the subalgebra spanned by the minimal ideals of the algebra.

Theorem 6. Let $A$ be a finite dimensional Bernstein algebra. Then $F(A)$ is an ideal.

Proof. We suppose $F(A)$ is not an ideal. Then there exists $x \in F(A)$ and $y \in A$ such 
that $x y \notin F(A)$. That is, for some maximal subalgebra $M$ of $A, x y \notin M$. Clearly $M \neq \operatorname{Ker} w$ and therefore $M$ contains a nontrivial idempotent $e$ such that $A=$ $K e+U_{e}+V_{e}$, and $M=K e+U_{e}^{\prime}+V_{e}$ with $U_{e}^{\prime} \leqq U_{e}$ such that $\operatorname{dim} U_{e}^{\prime}+1=\operatorname{dim} U_{e}$ because of Proposition 2.

In [5] it is shown that $F(A) \leqq(\operatorname{Ker} w)^{2}$ and then $x=u_{1}+u_{2}+v^{\prime}$ with $u_{1} \in U_{e} V_{e}$, $u_{2} \in V_{e}^{2}, v^{\prime} \in U_{e}^{2}$. Since $U_{e}^{2}$ and $V_{e}^{2} \leqq M$ because of Lemma 1 , it follows that $u_{1}, u_{2}, v^{\prime} \in M$. On the other hand $y=\lambda e+u+v$ with $\lambda \in K, u \in U_{e}, v \in V_{e}$. That is, $\lambda e$ and $v \in M$.

Thus $x y \notin M$ implies $u v^{\prime} \notin M$. But we can prove that if $v^{\prime} \in U_{e}^{2}$ and $u \in U_{e}-M$, then $u v^{\prime} \in M$. We suppose that $v^{\prime}=u^{\prime} u^{\prime \prime}$, with $u^{\prime}, u^{\prime \prime} \in U_{e}$. Since $A=M+K u$, then $u^{\prime}=a+\delta u$ and $u^{\prime \prime}=b+\omega u$ with $\delta, \omega \in K$ and $a, b, \in U_{e}^{\prime}$. Therefore

$$
u v^{\prime}=u\left(u^{\prime} u^{\prime \prime}\right)=u((a+\delta u)(b+\omega u))=u(a b)+\omega u(a u)+\delta u(u b)+\delta \omega u^{3} .
$$

But linearizing the first identity in (2) we have

$$
\begin{aligned}
& u(a b)=-a(u b)-b(u a) \in U_{e}^{\prime} U_{e}^{2} \leqq M \\
& u(a u)=-1 / 2 a u^{2} \in U_{e}^{\prime} U_{e}^{2} \leqq M \\
& u u^{2}=0 .
\end{aligned}
$$

Therefore $u v^{\prime} \in M$, which is a contradiction, and thus $x y \in F(A)$ and $F(A)$ is an ideal.

Proposition 7. Let $A$ be a Bernstein agelbra, and $e$ a nonzero idempotent of $A$ such that $A=K e+U_{e}+V_{e}$. Let $N=U_{e}+U_{e}^{2}$. Then $N^{2} \leqq F(A) \leqq(\operatorname{Ker} w)^{2}$.

Proof. In [5] we proved that $(\operatorname{Ker} w)^{2}$ contains $F(A)$.

On the other hand from [4] it is known that a Bernstein algebra $B$ with $B^{2}=B$ is genetic. It is easy to check that $B=K e+U_{e}+U_{e}^{2}$ satisfies this condition. Thus $B$ is genetic and from Corollary 5 we have $N^{2}=F(B)$. But $N$ is an ideal of $A$ and because of [7] (or checking it directly) $N^{2}$ is also an ideal of $A$. Now we apply (P1) and we have $N^{2} \leqq F(A)$.

Remark 8. Since $F(A) \leqq(\operatorname{Ker} w)^{2} \leqq U_{e}+U_{e}^{2}$ and $U_{e}+U_{e}^{2}$ is nilpotent, because $B=$ $\mathrm{Ke}+U_{e}+U_{e}^{2}$ is a genetic algebra, we have that $F(A)$ is nilpotent. (The author is aware that this result has also been obtained by $\mathrm{A}$. Koulibaly and $\mathrm{M}$. Ouattara).

Now we can consider the algebra $A / F(A)$, which is also a Bernstein algebra. From (P2) this algebra is such that $F(A / F(A))=F(A) / F(A)=0$. In the following we study Bernstein algebras such that $F(A)=0$. First we define two concepts: The zero socle of $A$, denoted Zsoc $(A)$, which is the sum of all minimal ideals with product zero and the socle of $A$, denoted by $\operatorname{Soc}(A)$, which is the sum of all minimal ideals of $A$. It is clear that Zsoc $(A) \leqq \operatorname{Soc}(A)$. In general for an arbitrary algebra Zsoc $(A) \neq \operatorname{Soc}(A)$, but in nontrivial Bernstein algebras Soc $(A)=Z \operatorname{Zsoc}(A)$. 
Proposition 9. Let $A$ be a Bernstein algebra such that if $e$ is nonzero idempotent $U_{e} \neq 0$. Then Zsoc $(A)=\operatorname{Soc}(A) \leqq \operatorname{Ker} w$.

Proof. We are going to prove that if $I$ is a minimal ideal of $A$ then $I$ has product zero and thus Zsoc $(A)=\operatorname{Soc}(A)$. Let $I$ be a minimal ideal of $A$. If $I$ is not contained in Ker $w$, then there exists a nonzero idempotent, $e$, in $I$ such that $A=K e+U_{e}+V_{e}$ and $I=K e+U_{e}^{\prime}+V_{e}^{\prime}$ with $U_{e}^{\prime} \leqq U_{e}$ and $V_{e}^{\prime} \leqq V_{e}$. But $U_{e}^{\prime}+V_{e}^{\prime}$ is an ideal of $A$ and $U_{e}^{\prime}+V_{e}^{\prime}$ is contained in $I$ and is different from $I$. Therefore $I \leqq \operatorname{Ker} w$ for every minimal ideal $I$ of $A$. From [7] we know that the product of ideals of $A$ contained in Ker $w$ is also an ideal of $A$. Thus $I^{2}=I$ or $I^{2}=0$. If $I^{2}=I$, then $K e+I=C$ is a Bernstein algebra such that $C^{2}=C$. That is, from [4], $C$ is a genetic algebra and therefore $I$ is nilpotent, which is a contradiction.

Theorem 10. Let $A$ be a Bernstein algebra such that $F(A)=0$. Then if $e$ is a nonzero idempotent of $A$ such that $A=K e+U_{e}+V_{e}$ we have:

(i) $U_{e}^{2}=0$,

(ii) Zsoc $(A)=U_{e}^{\prime}+V_{e}^{\prime}$ with $V_{e}^{\prime} \leqq V_{e}$ and $U_{e}^{\prime} \leqq U_{e}$ such that $V_{e}^{\prime} V_{e}=V_{e}^{\prime} U_{e}^{\prime}=0$,

(iii) $A=Z \operatorname{soc}(A)+C$ with $C$ a subalgebra of $A, C=(e+u)+W$ with $W \leqq\{-2 u v+$ $\left.v / v \in V_{e}\right\}$ such that $W^{2}=0$ and $u \in U_{e}$.

Moreover if $A$ is a Bernstein algebra verifying (i), (ii) and (iii) we have that $F(A)=0$.

Proof. Because of Proposition 7 we have (i).

For the proof of (ii), we consider a minimal ideal $I c Z \operatorname{Zsoc}(A)$. Let $e$ be a nonzero idempotent in $A$. Since $e I \leqq I$, we have that $I=\bar{U}_{e}+\bar{V}_{e}$ with $\bar{U}_{e} \leqq U_{e}$ and $\bar{V}_{e} \leqq V_{e}$. But from (i) $\bar{U}_{e} \cdot U_{e}=0$ and since $I$ is an ideal, $\bar{U}_{e} \cdot V_{e} \leqq U_{e} \cap I=\bar{U}_{e}$. Thus $\bar{U}_{e}$ is an ideal of $A$ and it is contained in the minimal ideal $I$ of $A$. Therefore we have $I=\bar{U}_{e}$ or $\bar{U}_{e}=0$. If $\bar{U}_{e}=0$, then $I=\bar{V}_{e}$. However $\bar{V}_{e} \cdot V_{e}$ and $\bar{V}_{e} \cdot U_{e}$ are contained in $\bar{U}_{e}$ and thus $\bar{V}_{e} \cdot A=0$. So Zsoc $(A)=U_{e}^{\prime}+V_{e}^{\prime}$ with $\left(U_{e}^{\prime}\right)^{2}=0=V_{e}^{\prime} U_{e}=V_{e} . V_{e}^{\prime}$. We remark that these conclusions follow for every nonzero idempotent using only the hypothesis $U_{e}^{2}=0$. Now from (P3) we have $A=Z \operatorname{soc}(A) \oplus C$ with $C$ a subalgebra of $A$. So $C$ contains a nonzero idempotent $e_{1}=e+u$ with $u \in U_{e}$ and $C=K e_{1} \oplus \tilde{U}_{e_{1}} \oplus W$ such that $\tilde{U}_{e_{1}} \leqq U_{e_{1}}$ and $W \leqq V_{e_{1}}$. We know that $U_{e_{1}}=\left\{u_{1}+2 u u_{1} / u_{1} \in U_{e}\right\}$ and $V_{e_{1}}=\left\{-2\left(u+u^{2}\right) v_{1}+v_{1} / v_{1} \in V_{e}\right\}$. Therefore $U_{e_{1}}=U_{e}$ and $V_{e_{1}}=\left\{-2 u v_{1}+v_{1} / v_{1} \in V_{e}\right\}$. Since $C \cap Z \operatorname{Zsoc}(A)=0$, we have that $C$ contains no minimal ideals of $A$. But $\tilde{U}_{e_{1}}$ is an ideal because $U_{e_{1}}=U_{e}, U_{e}^{2}=0, C$ is a subalgebra and $V_{e}^{\prime} \cdot U_{e}=0$. Therefore $\widetilde{U}_{e}=0$. Moreover if $C$ is a subalgebra, $W^{2} \leqq$ $U_{e} \cap C=0$.

Conversely let $A$ be a Bernstein algebra satisfying (i), (ii) and (iii). We know that $F(A) \leqq(\operatorname{Ker} w)^{2}=U_{e}^{2}+V_{e}^{2}+U_{e} V_{e}$. Thus $F(A) \leqq U_{e} W$ because of (i), (ii) and (iii). To prove (ii), note that we have shown using only (i), that $Z \operatorname{soc}(A)=U_{e}^{\prime}+V_{e}^{\prime}$ with $U_{e}^{\prime}=\sum_{i \in J} I_{i}$ and $V_{e}^{\prime}=\sum_{b \in B} J_{b}$ where $I_{i}, J_{b}$ are the minimal ideals of $A$ satisfying $I_{i} \leqq U_{e}$, and $I_{i}^{2}=0$ for all $i \in J$, and $J_{b} \leqq V_{e}, J_{b}^{2}=0$ for all $b \in B$. That is, the sums $\sum_{i \in J} I_{i}$ and $\sum_{b \in B} J_{b}$ are direct sums of algebras. Now we consider 


$$
A_{i}=\left(\sum_{s \in J s \neq i} I_{s}+V_{e}^{\prime}\right) \oplus C \text { and } D_{b}=\left(U_{e}^{\prime}+\sum_{s \in B s \neq b} J_{b}\right) \oplus C
$$

$A_{i}$ is ideal and $A / A_{i} \cong I_{i}$ is a nilpotent algebra. Moreover $D_{b}$ is also an ideal and $A / D_{b} \cong J_{b}$ is a nilpotent algebra. From (P4) we have $F\left(A / A_{i}\right)=\left(A / A_{i}\right)^{2} \cong I_{i}^{2}=0$ and $F\left(A / D_{b}\right)=\left(A / D_{b}\right)^{2} \cong J_{b}^{2}=0$. From (P2) we know that $\left(F(A)+A_{i}\right) / A_{i} \leqq F\left(A / A_{i}\right)=0$ for all $i \in J$ and $\left(F(A)+D_{b}\right) / D_{b} \leqq F\left(A / D_{b}\right)=0$ for all $b$ in $J$. So $F(A) \leqq\left(\bigcap A_{i}\right) \cap\left(\bigcap D_{b}\right) \leqq C$. But we also have $F(A) \leqq U_{e} . W \leqq U_{e}$. Therefore $F(A)=0$.

Corollary 11. Let $A$ be a Bernstein algebra such that $F(A)=0$. Then there exists a nonzero idempotent $e$ such that $A=\mathrm{Zsoc} A \oplus C$ with $Z s o c A=U_{e}^{\prime}+V_{e}^{\prime}, C=K e+\widetilde{V}_{e}$ and $V_{e}^{\prime}, \tilde{V}_{e} \leqq V_{e}$ verifying $V_{e}^{\prime} \cdot \widetilde{V}_{e}=0$.

Proof. From Theorem 10 and its proof we know that $A=\operatorname{Zsoc} A \oplus C$, where $C$ contains a nonzero idempotent $e, C=K e+\tilde{V}_{e}$ with $\tilde{V}_{e} \leqq V_{e}$ and Zsoc $A=U_{e}+V_{e}^{\prime}$ with $U_{e}^{\prime} \leqq U_{e}$ and $V_{e}^{\prime} \leqq V_{e}$ such that $V_{e}^{\prime} V_{e}=0$. Therefore $U_{e}^{\prime}=U_{e}$ and we have the corollary.

Acknowledgement. The author would like to express his gratitude to Professor Santos González of the University of Zaragoza for the supervision of this paper and the information he gave to him of another recent result about this subject.

\section{REFERENCES}

1. T. ConTÉs, Bernstein algebras: Lattice isomorphisms and isomorphisms (Nova Science Publishers), to appear.

2. I. M. H. Etherington, Genetic algebras, Proc. Roy. Soc. Edinburgh 59 (1939), 242-258.

3. I. M. H. Etherington, Duplication of linear algebras, Proc. Edinburgh Math. Soc. (2) 6 (1941), 222-230.

4. A. N. Grishkov, On the Genetic Property of Bernstein algebras, Soviet Math. Dokl. 35 (1987), 489-492.

5. J. Laliena, Maximal subalgebras of Bernstein algebras, in Clifford and Bernstein algebrasApplications (Université des Sciences et Techniques du Languedoc, Montpellier (France), 1989).

6. D. Towers, A Frattini theory for algebras, Proc. London Math. Soc. (3) 27 (1973), 440-462.

7. S. WALChER, Bernstein algebras which are Jordan algebras, Arch. Math. 50 (1988), 218-222.

8. A. Wörz-Busekros, Algebras in Genetics (Springer-Verlag. Lecture Notes in Biomathematics 36, Berlin Heidelberg 1980).

9. A. Wörz-Busekros, Bernstein algebras, Arch. Math. 48, (1987), 388-398.

Colegio Universitario de la Rioja

C/Obispo Bustamante 3

26001--LOGROÑo

Spain 